TITLE:

\title{
Random lasers with coherent feedback from highly transparent polymer films embedded with silver nanoparticles
}

\section{$\operatorname{AUTHOR}(\mathrm{S}):$}

Meng, Xiangeng; Fujita, Koji; Zong, Yanhua; Murai, Shunsuke; Tanaka, Katsuhisa

\section{CITATION:}

Meng, Xiangeng ... [et al]. Random lasers with coherent feedback from highly transparent polymer films embedded with silver nanoparticles. APPLIED PHYSICS LETTERS 2008, 92(20): 201112.

\section{ISSUE DATE:}

2008-05-19

URL:

http://hdl.handle.net/2433/84596

\section{RIGHT:}

Copyright 2008 American Institute of Physics. This article may be downloaded for personal use only. Any other use requires prior permission of the author and the American Institute of Physics. 


\title{
Random lasers with coherent feedback from highly transparent polymer films embedded with silver nanoparticles
}

\author{
Xiangeng Meng, ${ }^{1}$ Koji Fujita, ${ }^{1,2, a)}$ Yanhua Zong, ${ }^{1}$ Shunsuke Murai, ${ }^{1}$ and Katsuhisa Tanaka ${ }^{1}$ \\ ${ }^{1}$ Department of Material Chemistry, Graduate School of Engineering, Kyoto University, Katsura, \\ Nishikyo-ku 615-8510, Kyoto, Japan \\ ${ }^{2}$ PRESTO, Japan Science and Technology Agency (JST), 4-1-8, Honcho Kawaguchi, \\ Saitama 332-0012, Japan
}

(Received 15 March 2008; accepted 31 March 2008; published online 23 May 2008)

\begin{abstract}
We report on observations of random lasers with coherent feedback in highly transparent polymer films embedded with silver nanoparticles. The hybrid materials were fabricated via in situ synthesis method, through which silver nanoparticles were precipitated by thermal treatment. Sharp peaks with linewidth $\sim 0.5 \mathrm{~nm}$ were observed to emerge on the broad emission background when the pump energy reached a threshold, together with unidirectional laser irradiation. Random lasers with coherent feedback induced by silver nanoparticles have been rarely reported, hence, we expect that this work will add an aspect to random lasers by using metal nanostructures to obtain coherent feedback. (C) 2008 American Institute of Physics. [DOI: 10.1063/1.2912527]
\end{abstract}

Random lasers have attracted tremendous attention since they theoretically concern the fundamental physics of Anderson localization for photon and experimentally provide potential laser devices readily accessible from a technical point of view. ${ }^{1-6}$ Random lasers operate based on light scattering, that is why the scattering strength of random media is crucial to evaluate whether a coherent feedback is involved or not. Generally, weak scattering strength results in a gradual narrowing of emission spectrum with linewidth in scale of several nanometers while strong scattering strength gives rise to discrete sharp peaks with linewidth less than $1 \mathrm{~nm}$ superimposed on the emission spectrum. In order to achieve coherent feedback, dielectric materials with high refractive index (e.g., $\mathrm{GaP}, \mathrm{TiO}_{2}$, and $\mathrm{ZnO}$ ) have been utilized for several decades because large refractive index contrast significantly contributes to the intense scattering according to the Mie scattering theory. ${ }^{7-9}$

Metal nanoparticles are potential candidates that may play an important role in coherent feedback in random lasers. The potential of metal nanoparticles toward applications in random lasers includes two aspects. First of all, metal nanoparticles have much larger scattering cross section $\left(\sigma_{s}\right)$ than dielectric nanoparticles with the same dimensions. Second, metal nanoparticles are more advantageous in their unique surface plasmon resonance (SPR) property, which has proved to spatially confine light wave near particle surface so that high gain for lasing is expected. Unfortunately, metal nanoparticles toward random lasers suffer from light absorption that is detrimental to laser oscillation and negligible for dielectric nanoparticles-based random lasers. This may explain why coherent feedback random lasers have not been observed in media composed of metal nanoparticles. ${ }^{10,11}$

In this letter, we report on a breakthrough in metal nanoparticles-based random lasers: random lasers with coherent feedback have been observed in polymer films embedded with silver nanoparticles. Silver nanoparticles embedded inside polymer films were prepared via in situ reduction. ${ }^{12}$ In the procedure, the mixture of polyvinyl alco-

${ }^{a)}$ Electronic mail: fujita@dipole7.kuic.kyoto-u.ac.jp. hol (PVA), silver nitrate, and rhodamine 6G (R6G) aqueous solutions is spin coated on glass substrate followed by annealing, leading to the precipitation of silver nanoparticles. The evolution of silver nanoparticles in PVA matrix against annealing time was characterized by optical absorption spectra recorded with a JASCO-V570 ultraviolet-visible-near infrared spectrophotometer. The samples were optically pumped by the second harmonics of a mode-locked Nd: yttrium aluminum garnet (YAG) laser $(\lambda=1064 \mathrm{~nm}, 10 \mathrm{~Hz}$ repetition rate, 25 ps pulse duration) in order to conduct random laser experiments. The laser beam was focused on the samples through a cylindrical lens to form a stripe with a width of $17 \mu \mathrm{m}$ and a length that could be varied from 0.1 to $6.0 \mathrm{~mm}$. Figure 1 shows the configuration of PVA-AgR6G film and excitation-detection. The emission was collected along the stripe with a fiber device into a monochromator (SPEX 270M) coupled to a liquid nitrogen cooled charge couple device (CCD 3000, the spectral resolution is $0.07 \mathrm{~nm})$.

Figure 2(a) describes the evolution of optical absorption spectra for PVA-Ag-R6G films as a function of annealing time at $110^{\circ} \mathrm{C}$. An absorption band at around $\lambda=420 \mathrm{~nm}$

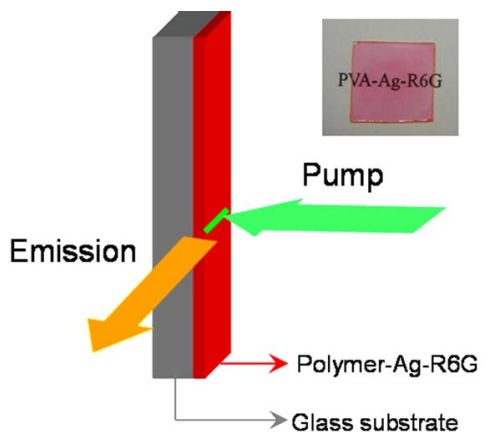

FIG. 1. (Color online) Schematic illustration of configuration of PVA-AgR6G film and excitation-detection. The PVA acts as a matrix while silver nanoparticles as scattering centers and R6G as a light emitting material. The film thickness is about $4 \mu \mathrm{m}$. The sample is pumped by the second harmonics of a mode-locked Nd:YAG laser $(\lambda=1064 \mathrm{~nm}, 10 \mathrm{~Hz}$ repetition rate, $25 \mathrm{ps}$ pulse duration). The inset is a photograph of the sample (top view). 

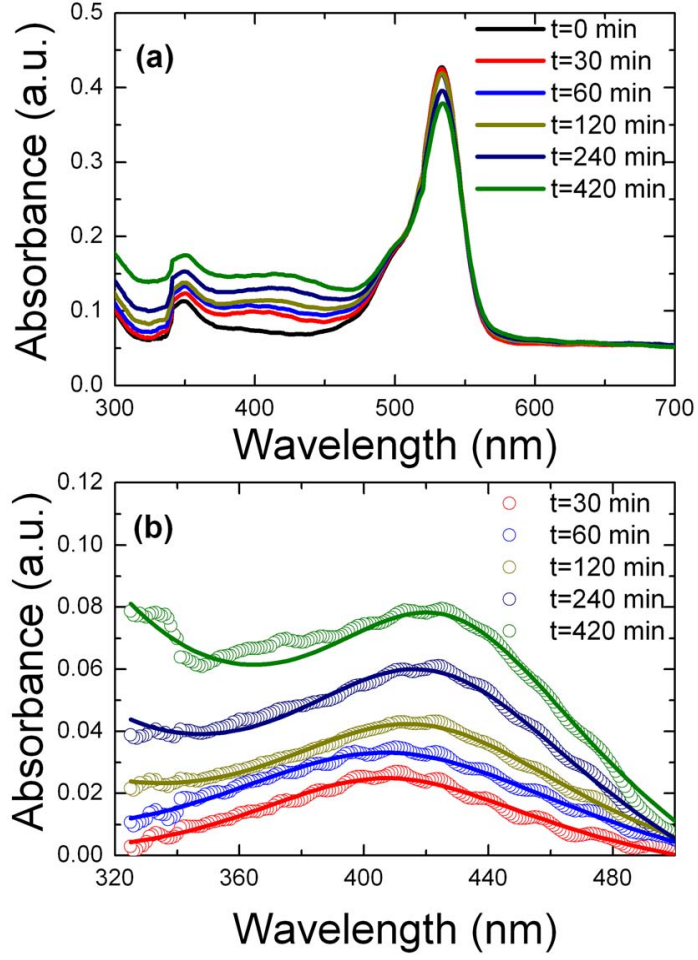

FIG. 2. (Color online) (a) Optical absorption spectra of PVA-Ag-R6G film as a function of annealing times: $t=0$ (black), 30 (red), 60 (blue), 120 (dark yellow), 240 (navy), and $420 \mathrm{~min}$ (olive). (b) The difference spectra (open circles) between the annealed samples and as-prepared one along with the simulation (solid lines) by the Mie scattering theory and Drude model. The sample was annealed at $110^{\circ} \mathrm{C}$.

emerged when the annealing time reached $30 \mathrm{~min}$ and then gradually rose with the increase in annealing time and finally saturated after $420 \mathrm{~min}$ annealing. This band can be ascribed to SPR signal of spherical silver nanocrystals. During the thermal treatment, the PVA reduces $\mathrm{Ag}^{+}$ion while the alcohol is oxidized to ketone. ${ }^{13}$ The absorption band at around $\lambda=530 \mathrm{~nm}$ is due to the electronic transition from the ground state $S_{0}$ to the first singlet excited state $S_{1}$ of R6G. Figure 2(b) presents the difference in absorption spectra between annealed samples and as-prepared one, as well as the simulation curves by using the Mie scattering theory and Drude model. ${ }^{14}$ The curves at longer wavelength are not involved in the simulation process since they are significantly affected by the absorption band at around $\lambda=530 \mathrm{~nm}$ of R6G. The evolution of SPR signal is very obvious with an increase in the annealing time. The simulation result indicates that the average size of $\mathrm{Ag}$ nanoparticles is $\sim 1.0 \mathrm{~nm}$ in radius while the volume fraction of silver nanoparticles relative to PVA is $\sim 3.8 \times 10^{-4}$. The particle size is consistent with the previous report. ${ }^{12}$ It should be noted that no change of appearance has been found in the sample during the $532 \mathrm{~nm}$ laser irradiation in laser experiment, indicating that the pump laser has no influence on silver nanoparticles in PVA matrix.

Figure 3 depicts the evolution of emission spectra as a function of pump energy. The emission spectrum shows a broad spontaneous emission band with a linewidth of $\sim 32 \mathrm{~nm}$ when the pump energy is low. However, when the pump energy reaches the threshold (herein $\sim 1.12 \mu \mathrm{J}$ ), a sharp peak that centers at $\lambda \sim 567 \mathrm{~nm}$ with a linewidth of $\sim 0.5 \mathrm{~nm}$ suddenly emerges on the broad amplified spontaneous emission background. In contrast, the sample contain-

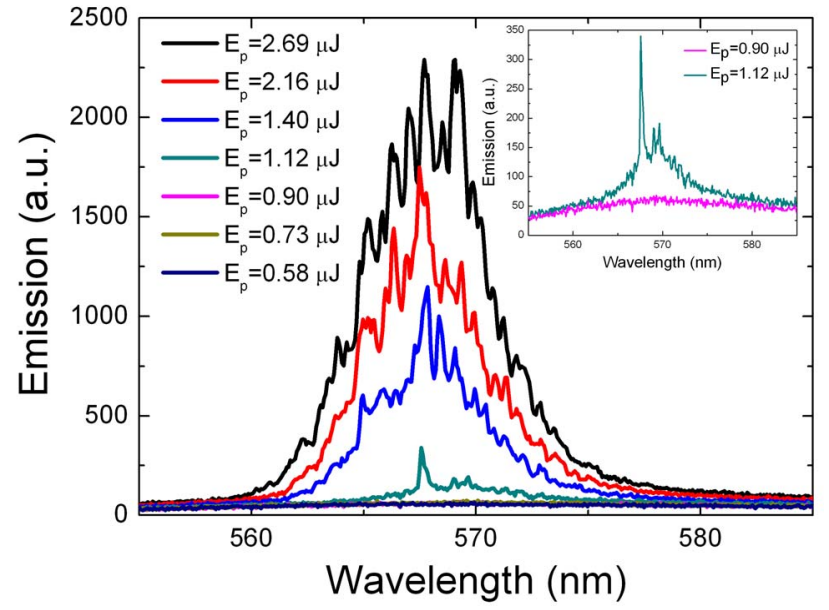

FIG. 3. (Color online) Emission spectra as a function of pump energy for PVA-Ag-R6G film. The inset shows the emission spectra just below and above the pump threshold. The stripe width and length is $17 \mu \mathrm{m}$ and $2.4 \mathrm{~mm}$, respectively.

ing no silver nanoparticles merely showed a spontaneous emission band, indicating that silver nanoparticles have played a crucial role in the emergence of sharp peaks. With a further increase in pump energy, more sharp peaks are observed in the broad emission background. For a clear demonstration of sharp peaks, the emission spectra just below and above the pump threshold are inserted in Fig. 3. We suppose that these peaks are really due to laser oscillation by analyzing the evolution of emission intensity as a function of pump energy that clearly confirms a threshold behavior, as shown in Fig. 4. Another evidence for laser oscillation is the observation of unidirectional laser emission, as shown in the inset of Fig. 4, since the spontaneous emission is lack of unidirectional irradiation. The results in Fig. 4 resemble reported random lasers with coherent feedback. ${ }^{5,9}$ For instance, the number of laser modes is small when the pump energy is relatively low since only those cavities with gain high enough can lead to lasing. The increase in pump energy increases the number of cavities that contribute to the lasing.

The extinction cross section $\left(\sigma_{e}\right)$ of one particle consists the scattering and absorption cross section $\left(\sigma_{a}\right)$, namely, $\sigma_{e}=\sigma_{s}+\sigma_{a}$. The scattering cross section of one $\mathrm{Ag}$

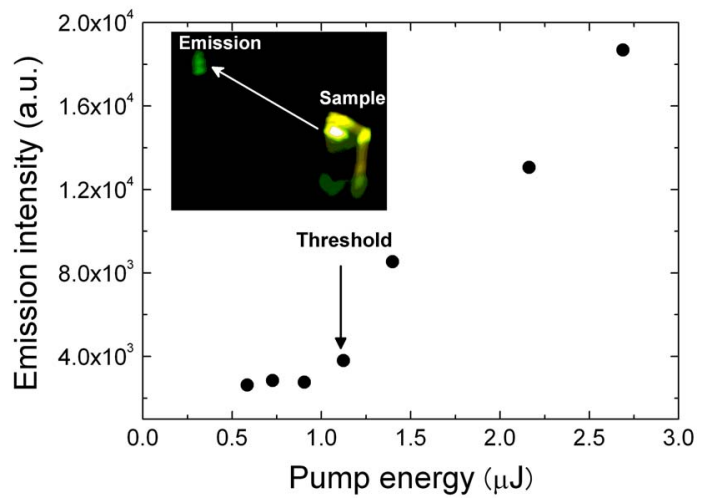

FIG. 4. (Color online) Evolution of emission intensity as a function of pump energy. The transition from spontaneous emission to laser irradiation is clearly indicated by the sharp increase in the slope. The inset is a photograph of emission when the pump energy is above threshold. At right is the sample while the left is the emission projected on a screen $12 \mathrm{~cm}$ away from the sample edge. 
nanosphere with $1.0 \mathrm{~nm}$ in radius is $\sigma_{s} \sim 1.5 \times 10^{-24} \mathrm{~m}^{2}$ while $\sigma_{a} \sim 5.3 \times 10^{-21} \mathrm{~m}^{2}$ at $\lambda=567 \mathrm{~nm}$. Therefore, the scattering intensity of such a silver nanoparticle is much weaker than the absorption intensity. However, we have found certain enhancement of fluorescence instead of fluorescence quenching for all the samples containing silver nanoparticles. The results indicate that the enhancement is possibly caused by SPR, as previously revealed. ${ }^{15}$ The scattering mean free path $l_{s}$ can be estimated via the Mie theory $l_{s}=1 / \rho \sigma_{s}$ where $\rho$ is the number density of silver nanoparticles in PVA host. The $\rho$ is calculated to be $\sim 9.1 \times 10^{22} / \mathrm{m}^{3}$, thus, $l_{s} \sim 7.3$ $\times 10^{3} \mathrm{~mm}$. Therefore, $k l_{s} \sim 1.2 \times 10^{8} \gg 1$, implying that the present system operates in extremely weak scattering regime. Although coherent feedback random lasers have been observed in dielectric based random media, ${ }^{16,17}$ the scattering strength as weak as that in this work has never been conducted. We surmise that the observed lasing peaks are associated with the highly localized optical modes due to SPR.

The present random media include three important characteristics that make it distinct from existing random media. First, metal nanoparticles instead of dielectric nanoparticles are used as scattering centers. Second, the particle size is much smaller than the emission wavelength, which rules out the possibility of single particle as a resonator. Third, the scattering strength is extremely weak, which makes polymer films highly transparent for efficient optical pumping. Furthermore, the number density and particle size of silver nanoparticles in PVA matrix can be facilely manipulated through annealing temperature and time, as well as the initial concentration of $\mathrm{Ag}^{+}$ions.

In summary, we have observed random lasers with coherent feedback in highly transparent polymer films embedded with silver nanoparticles. The scattering mean free path estimated by the Mie theory is about eight orders of magnitude larger than the emission wavelength, indicating that normal light scattering barely contributes to the emergence of coherent feedback. We infer that the observed random lasers with coherent feedback are closely associated with highly localized light modes caused by SPR interaction between silver nanoparticles and light. We expect that this work adds an aspect to the fields of random lasers with metal nanostructure-based random media.

This study was financially supported by the Grand in Aid for Scientific Research (No. 18360316) from the Ministry of Education, Culture, Sport, Science, and Technology (MEXT), Japan.

${ }^{1}$ V. S. Letokhov, Zh. Eksp. Teor. Fiz. 53, 1442 (1967); Sov. Phys. JETP 26, 835 (1968).

${ }^{2}$ V. M. Markushev, V. F. Zolin, and Ch. M. Briskina, Zh. Prikl. Spektrosk. 45, 847 (1986); N. E. Ter-Gabrielyan, V. M. Markushev, V. R. Belan, Ch. M. Briskina, O. V. Dimitrova, V. F. Zolin, and A. V. Lavrov, Sov. J. Quantum Electron. 21, 840 (1991).

${ }^{3}$ N. M. Lawandy, R. M. Balachandran, A. S. L. Gomes, and E. Sauvain, Nature (London) 368, 436 (1994).

${ }^{4}$ D. S. Wiersma and A. Lagendijk, Phys. Rev. E 54, 4256 (1996).

${ }^{5}$ H. Cao, Y. G. Zhao, S. T. Ho, E. W. Seeling, Q. H. Wang, and R. P. H. Chang, Phys. Rev. Lett. 82, 2278 (1999).

${ }^{6}$ S. V. Frolov, W. Gellerman, M. Ozaki, K. Yoshino, and Z. V. Vardeny, Phys. Rev. Lett. 78, 729 (1997).

${ }^{7}$ K. L. van der Molen, R. W. Tjerkstra, A. P. Mosk, and A. Lagendijk, Phys. Rev. Lett. 98, 143901 (2007).

${ }^{8}$ G. van Soest, F. J. Poelwijk, R. Sprik, and A. Lagendijk, Phys. Rev. Lett. 86, 1522 (2001).

${ }^{9}$ S. F. Yu and E. S. P. Leong, IEEE J. Quantum Electron. 40, 1186 (2004).

${ }^{10}$ G. D. Dice, S. Mujumdamar, and A. Y. Elezzabi, Appl. Phys. Lett. 86, 131105 (2005).

${ }^{11}$ O. Popov, A. Zilbershtein, and D. Davidov, Appl. Phys. Lett. 89, 191116 (2006).

${ }^{12}$ S. Porel, S. Singh, S. S. Harsha, D. N. Rao, and T. P. Radhakrishnan, Chem. Mater. 17, 9 (2005).

${ }^{13}$ S. Porel, S. Singh, and T. P. Radhakrishnan, Chem. Commun. (Cambridge) 2005, 2387.

${ }^{14}$ S. Murai, K. Fujita, S. Kawase, S. Ukon, and K. Tanaka, J. Appl. Phys. 102, 073515 (2007).

${ }^{15}$ K. Okamoto, I. Niki, A. Shvartser, Y. Narukawa, T. Mukai, and S. Scherer, Nat. Mater. 3, 601 (2004).

${ }^{16}$ R. C. Polson, A. Chipouline, and Z. V. Vardeny, Adv. Mater. (Weinheim, Ger.) 13, 760 (2001).

${ }^{17}$ X. Wu, W. Fang, A. Yamilov, A. A. Chabanov, A. A. Asatryan, L. C. Botten, and H. Cao, Phys. Rev. A 74, 053812 (2006). 